\title{
EFFECT OF PLATELET CONCENTRATION AND CALCIUM ON PLASMA CLOT ABSORBANCE
}

\author{
Modepalli Susree $^{1}$, Mohan Anand ${ }^{2}$ \\ ${ }^{1}$ Research Scholar, Department of Chemical Engineering, Indian Institute of Technology, Hyderabad, Telangana, \\ India \\ ${ }^{2}$ Associate Professor, Department of Chemical Engineering, Indian Institute of Technology, Hyderabad, Telangana, \\ India
}

\begin{abstract}
Clot structure is greatly influenced by a number of factors in vivo, calcium concentration being one of them. A pilot study with 10 healthy individuals was performed to investigate the effect of added calcium, in combination with plasma platelet concentration, on the turbidity of clots. Using a UV-Vis spectrophotometer, absorbance of both platelet-rich and platelet-poor clots was taken at $405 \mathrm{~nm}$, in the presence as well as the absence of added calcium. A 9.93\% increase in the maximum absorbance is observed for PRP with added calcium while a 7.68\% increase was observed for PPP. Also, PRP clots reported higher absorbance than PPP clots both with (16.48\%) and without (14.10\%) calcium. It was also observed in parallel that platelet concentration also affected turbidity of the clots with \& without added calcium.
\end{abstract}

Keywords: Calcium, Platelet-Poor Plasma (PPP), Platelet-Rich Plasma (PRP), Thrombin, and Turbidity.

\section{INTRODUCTION}

The ability of blood to coagulate and hence curb blood loss from the body depends on its capacity to generate the protein thrombin. Being the last enzyme produced by the coagulation cascade, thrombin catalyzes the conversion of fibrinogen to fibrin thereby forming a mesh and stabilizing the platelet aggregates. The coagulation cascade has been comprehended as a sequential occurrence of enzymatic events that include both pro and inhibitory reactions in order to avoid both hypercoagulation and excessive bleeding, respectively. A qualitative as well as quantitative analysis of the different sections of the enzymatic cascade of blood coagulation would provide clinicians with valuable insights into the coagulation potential of an individual or a population as a whole[1]. Upon correlation with clinical consequences, these tests can further quantitatively signify effects of different drugs on the individual or group of individuals [2].

Though complicated, a direct estimation of plasma fibrinogen is done by measuring protein of the fibrin clot. Structure of the fibrin clot has been widely studied using optical methods $[\mathbf{3}, \mathbf{4}, \mathbf{5}, \mathbf{6}, \mathbf{7}]$. One of the first accurate and simple optical methods for determining plasma fibrinogen, by measuring an increase in the absorbance as fibrinogen converts to fibrin, was developed by [8]. Investigation of the fibrin clot fiber structure presents potential clinical implications with respect to strength, stability and resistance of the clot to fibrinolysis. As a qualitative measure, an altered fibrin clot structure can be indicative of abnormal bleeding or hypercoagulability thus providing a correlation with an individual's thrombotic pathophysiology $[9,10,11$, 12].
It has been reported that fibrin fibers formed in plasma are more massive than those formed from purified fibrin; an increase in calcium concentration led to an increase in fiber size [13]. A lot of factors have been known to affect the structural properties of fibrin clot in plasma. The influence of thrombin generation [10], calcium [3], pH, ionic strength \& temperature $[\mathbf{1 4}, \mathbf{1 5}]$ have been investigated among others. These factors not only influence the fibrin monomer generation, polymerization, cross-linking but also have profound effect on fibrin fiber size \& thickness, network assembly and clotting time. [3] report fibrin clots (clotted with thrombin) with higher elastic moduli, and hence greater rigidity, in the presence of calcium ions. However, they also convey that the elasticity is irreversibly reduced upon removal of tightly bound calcium ions (by chelation with EDTA), even in the presence of added calcium. In addition, the calcium-dependent release of fibrinopeptides by thrombin at different concentrations of $\mathrm{CaCl}_{2}[\mathbf{1 6}]$, as well as the physiological role of calcium in the unfolding of fibrin monomers [17] have been studied and reported. Presence of both high-affinity and low-affinity calcium-binding sites on fibrinogen, which is essential for both polymerization \& stability of clots, has been ascertained $[\mathbf{1 8}, \mathbf{1 9}]$.

We present here a brief qualitative study of the effect of calcium on the plasma clot formation in vitro, and also with respect to platelet concentration in the plasma which has not been studied previously. The study investigates clotting of plasma (both platelet-rich \& platelet-poor) obtained from 10 different individuals by observing the progressive change in absorbance of the plasma for 300 seconds after the addition of fixed concentration of thrombin to platelet-rich as well as platelet-poor plasma in vitro, both with and without recalcification. 


\section{MATERIALS \& METHODS}

\subsection{Collection and Preparation of Plasma}

This study was conducted with the informed consent of volunteer subjects, and the experimental protocol was approved by the Institute Ethics Committee at IIT, Hyderabad. Ten healthy donors, whose ages ranged from 20 to 35 , were enrolled in this study. All donors were not on any medication, including aspirin and other nonsteroidal antiinflammatory drugs, during the 2 weeks before this study. Whole blood ( $9 \mathrm{~mL}$ ) was drawn by venipuncture and collected into vacutainer tubes (BD, India) that contained $3.2 \%$ citrate $(0.3 \mathrm{~mL})$ as an anticoagulant.

To isolate platelet-rich plasma, tubes were centrifuged at $200 \mathrm{~g}$ for 10 minutes at $20^{\circ} \mathrm{C}$. Half volume of the supernatant, containing the platelet-rich plasma was gently transferred to an empty tube and labeled appropriately. The remaining plasma was centrifuged again at a higher speed $\left(1000 \mathrm{~g}, 10 \mathrm{~min}, 20^{\circ} \mathrm{C}\right.$ ) to precipitate the platelets at the bottom of the tube. Platelet-poor plasma was thus obtained after the second spin; this was transferred to an empty tube and labeled appropriately.

\subsection{Preparation of Thrombin Solution}

High quality human plasma thrombin was obtained from Merck Millipore, India in a lyophillized form. The enzyme was reconstituted in distilled water according to its specific activity, as instructed by the supplier, to prepare stock solution. A working solution equivalent to $1 \mathrm{U}$ thrombin per $\mathrm{mL}$ of distilled water was prepared from the stock each time before use.

\subsection{Preparation of $\mathrm{CaCl}_{2} \&$ HBS}

$1 \mathrm{M} \mathrm{CaCl}_{2}$ was prepared by dissolving laboratory grade $\mathrm{CaCl}_{2}$ in distilled water.

HBS (HEPES-buffered saline) was prepared constituting of $20 \mathrm{mM}$ HEPES and $140 \mathrm{mM} \mathrm{NaCl}$. The $\mathrm{pH}$ of HBS solution was adjusted to 7.4 .

\subsection{UV-Vis Spectrophotometer Analysis}

The samples for reading the absorbance were directly prepared in the cuvette. Clotting was initiated by adding $1 \mathrm{U}$ thrombin to $100 \mu \mathrm{L}$ plasma diluted with $\operatorname{HBS}$ (1:5), and allowed to proceed at room temperature $\left(26^{\circ} \mathrm{C}\right)$. For samples clotting in the presence of calcium, $10 \mu \mathrm{L} 1 \mathrm{M} \mathrm{CaCl}_{2}$ was added before adding the enzyme. An initial reading was taken before addition of the enzyme.

The absorbance was read at $405 \mathrm{~nm}$ using a Perkin-Elmer UV-Vis Spectrophotometer once every $30 \mathrm{sec}$ for $5 \mathrm{~min}$ in a 1 $\mathrm{cm}$ path-length cell to track the change in turbidity of the sample.

HBS was used as a blank for the reference optical density measurement.

\section{RESULTS \& DISCUSSION}

Absorbance was taken for each of the four different samples that were prepared using the plasma obtained from each donor - PRP, PRP $+\mathrm{Ca}^{2+}, \mathrm{PPP}, \mathrm{PPP}+\mathrm{Ca}^{2+}$.

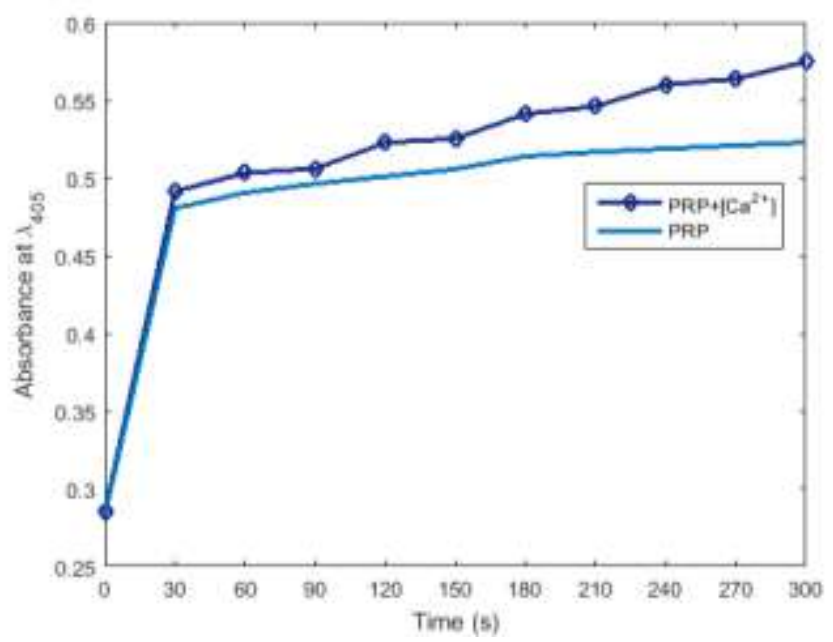

Fig -1: Effect of calcium on change in absorbance of PRP at $\lambda_{405}$.

Fig. 1 tracks the increase in absorbance as clotting proceeds in PRP, in the presence (plot with markers) as well in the absence of added calcium. A 9.93\% increase in the maximum absorbance is observed for PRP with added calcium.

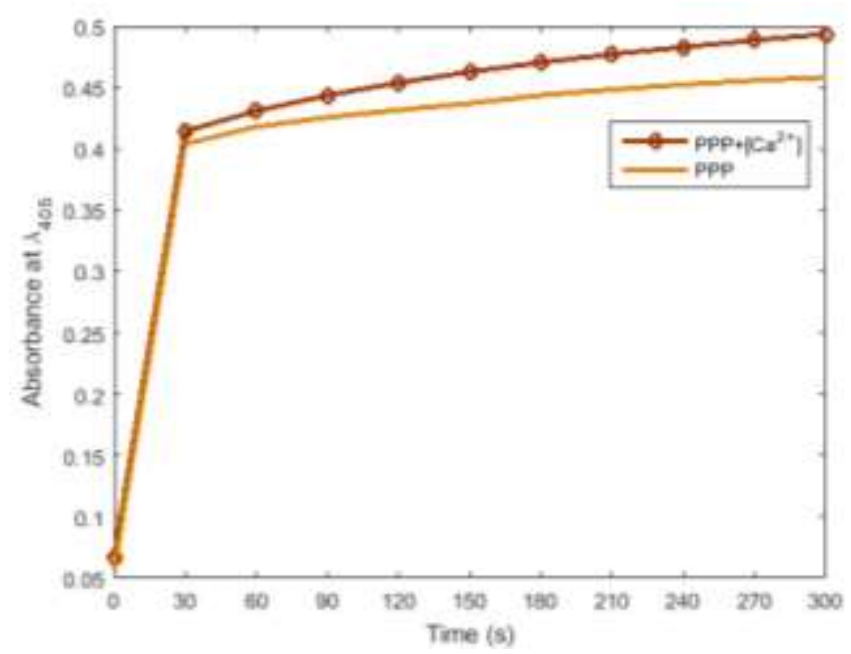

Fig -2: Effect of calcium on change in absorbance of PPP at $\lambda_{405}$.

Similarly for PPP, the change in absorbance in the presence (plot with markers) as well as absence of added calcium has been plotted in Fig. 2. There is a $7.68 \%$ increase in the maximum absorbance for PPP with added calcium.

In both cases, it was seen that the change in absorbance after 5 min was minimal.

Fine fibers (i.e, with low fiber diameter) are known to produce clots with less turbidity [6]. In both cases of PRP \& PPP clots, those formed in the presence of added calcium 
showed slightly higher turbidity while those without any added calcium were relatively transparent; this is also evident in the respective values of absorbance recorded for each clot type.

The relatively higher absorbance of PRP clots than that of PPP clots, in both cases (16.48\% with calcium, and $14.10 \%$ without calcium), is also noteworthy.

\section{CONCLUSIONS}

A pilot study was performed involving the participation of 10 healthy volunteers. Platelet-rich and platelet-poor clots were formed by adding thrombin to the prepared plasma samples under two different conditions: presence, and absence of added calcium. We have investigated the effect in both PRP \& PPP which has not been reported previously. We found that, in the presence of calcium, both platelet-rich and platelet-poor clots reported higher absorbance while in the absence of calcium the clots appeared nearly transparent. This can be attributed to the fact that calcium is crucial for assembly of procoagulant complexes and thrombin generation in vivo. As a result, clotting begins early thereby producing thicker fibrin fibers than are seen in the absence of calcium.

Simultaneously, we also observed that platelet-rich clots are more turbid than platelet-poor clots irrespective of the presence of added calcium.

\section{ACKNOWLEDGEMENT}

MS was supported by the MHRD Fellowship for Research Scholars administered by IIT Hyderabad. This research was funded by the institute seed grant given to MA at IIT Hyderabad.

\section{REFERENCES}

[1]. H. Hemker, S. Wielders, H. Kessels and S. Beguin, "Continuous registration of thrombin generation in plasma, its use for the determination of the thrombin potential", Thromb. Haemost., vol. 70, no. 4, pp. 6176241993.

[2]. T. Baglin, "The measurement and application of thrombin generation", Br. J. Haematol., vol. 130, no. 5, pp. 653-661 2005.

[3]. L. L. Shen, R. P. McDonagh, J. McDonagh and J. Hermans, "Fibrin gel structure: Influence of calcium and covalent cross-linking on the elasticity", Biochem. Biophys. Res. Commun., vol. 56, no. 3, pp. 793-798 1974.

[4]. Y. Inada, H. Okamoto, S. Kanai and Y. Tamaura, "Faster determination of clottable fibrinogen in human plasma: An improved method and kinetic study", Clin. Chem., vol. 24, no. 2, pp. 351-353 1978.

[5]. A. Wolberg, D. Gabriel and M. Hoffman, "Analyzing fibrin clot structure using a microplate reader", Blood Coagul. Fibrinolysis, vol. 13, p. 533-539 2002.

[6]. C. Yeromonahos, B. Polack and F. Caton, "Nanostructure of the fibrin clot", Biophys. J., vol. 99, no. 7, pp. 2018-2027 2010.
[7]. K. M. Weigandt, N. White, D. Chung, E. Ellingson, Y. Wang, X. Fu and D. C. Pozzo, "Fibrin clot structure and mechanics associated with specific oxidation of methionine residues in fibrinogen", Biophys. J., vol. 103, no. 11, pp. 2399-2407 2012.

[8]. B. Ellis and A. Stransky, "A quick and accurate method for the determination of fibronogen in plasma", The Journal of laboratory and clinical medicine, vol. 58, p. 4771961.

[9]. C. W. Francis, D. Connaghan, W. Scott and V. Marder, "Increased plasma concentration of cross-linked fibrin polymers in acute myocardial infarction", Circulation, vol. 75, no. 6, pp. 1170-1177 1987.

[10].A. S. Wolberg and R. A. Campbell, "Thrombin generation, fibrin clot formation and hemostasis", Transfus. Apher. Sci., vol. 38, no. 1, pp. 15-23 2008.

[11].A. Undas and R. A. Ariëns, "Fibrin clot structure and function a role in the pathophysiology of arterial and venous thromboembolic diseases", Arterioscler. Thromb. Vasc. Biol., vol. 31, no. 12, pp. e88-e99 2011.

[12].S. Neergaard-Petersen, A.-M. Hvas, R. Ajjan, S. B. Larsen, M. Würtz, S. D. Kristensen and E. L. Grove, "Platelet count, platelet turnover and fibrin clot structure in patients with coronary artery disease", Thromb. Res., vol. 133, no. 6, p. 11612014.

[13].M. E. Carr, "Fibrin formed in plasma is composed of fibers more massive than those formed from purified fibrinogen", Thromb. Haemost., vol. 59, no. 3, pp. 5355391988.

[14].C. Nair, G. Shah and D. Dhall, "Effect of temperature, ph and ionic strength and composition on fibrin network structure and its development", Thromb. Res., vol. 42, no. 6, pp. 809-816 1986.

[15].Z. S. Latallo, A. P. Fletcher, N. Alkjaersig and S. Sherry, "Influence of ph, ionic strength, neutral ions, and thrombin on fibrin polymerization", American Journal of Physiology--Legacy Content, vol. 202, no. 4, pp. 675-680 1962.

[16].A. Profumo, M. Turci, G. Damonte, F. Ferri, D. Magatti, B. Cardinali, C. Cuniberti and M. Rocco, "Kinetics of fibrinopeptide release by thrombin as a function of cacl 2 concentration: Different susceptibility of fpa and $\mathrm{fpb}$ and evidence for a fibrinogen isoformspecific effect at physiological ca2+ concentration", Biochemistry, vol. 42, no. 42, pp. 12335-12348 2003.

[17].L. E. Averett, B. B. Akhremitchev, M. H. Schoenfisch and O. V. Gorkun, "Calcium dependence of fibrin nanomechanics: The $\gamma 1$ calcium mediates the unfolding of fibrinogen induced by force applied to the " $\mathrm{a}-\mathrm{a}$ " bond", Langmuir, vol. 26, no. 18, pp. 14716-14722 2010.

[18].C. Dang, C. K. Shin, W. R. Bell, C. Nagaswami and J. Weisel, "Fibrinogen sialic acid residues are low affinity calcium-binding sites that influence fibrin assembly", $J$. Biol. Chem., vol. 264, no. 25, pp. 15104-15108 1989.

[19].J. W. Weisel and R. I. Litvinov, "Mechanisms of fibrin polymerization and clinical implications", Blood, vol. 121, no. 10, pp. 1712-1719 2013. 